A LETTERS JOURNAL EXPLORING THE FRONTIERS OF PHYSICS

\title{
OFFPRINT
}

\section{Unstable dynamical systems: Delays, noise and control}

\author{
J. G. Milton, J. L. Cabrera and T. Ohira \\ EPL, 83 (2008) 48001
}

Please visit the new website

www.epljournal.org 


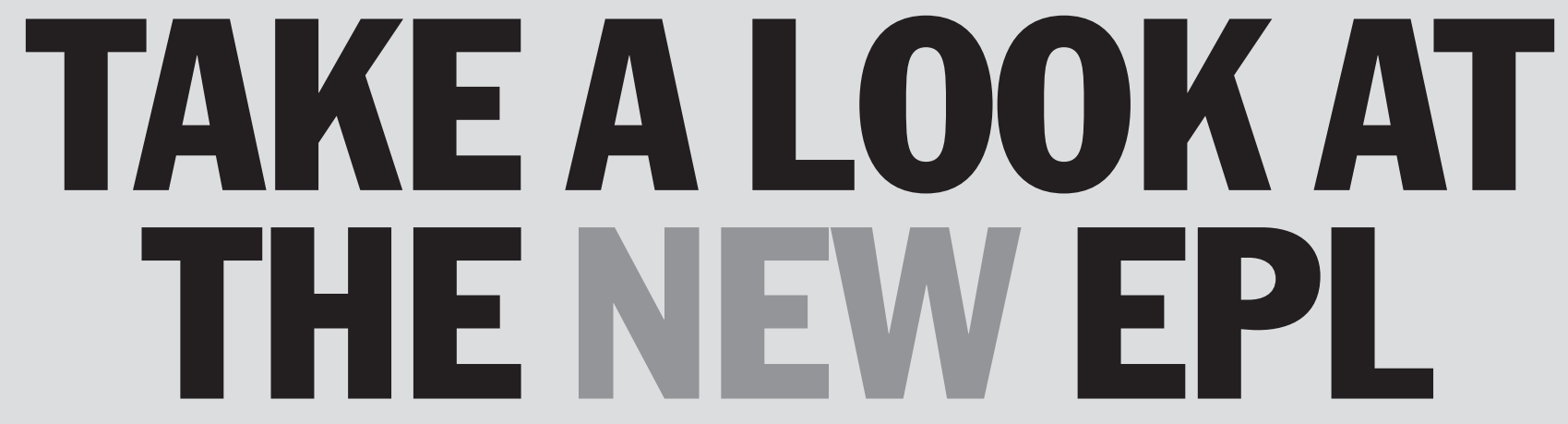

\section{Europhysics Letters (EPL) has a new online home at www.epljournal.org}

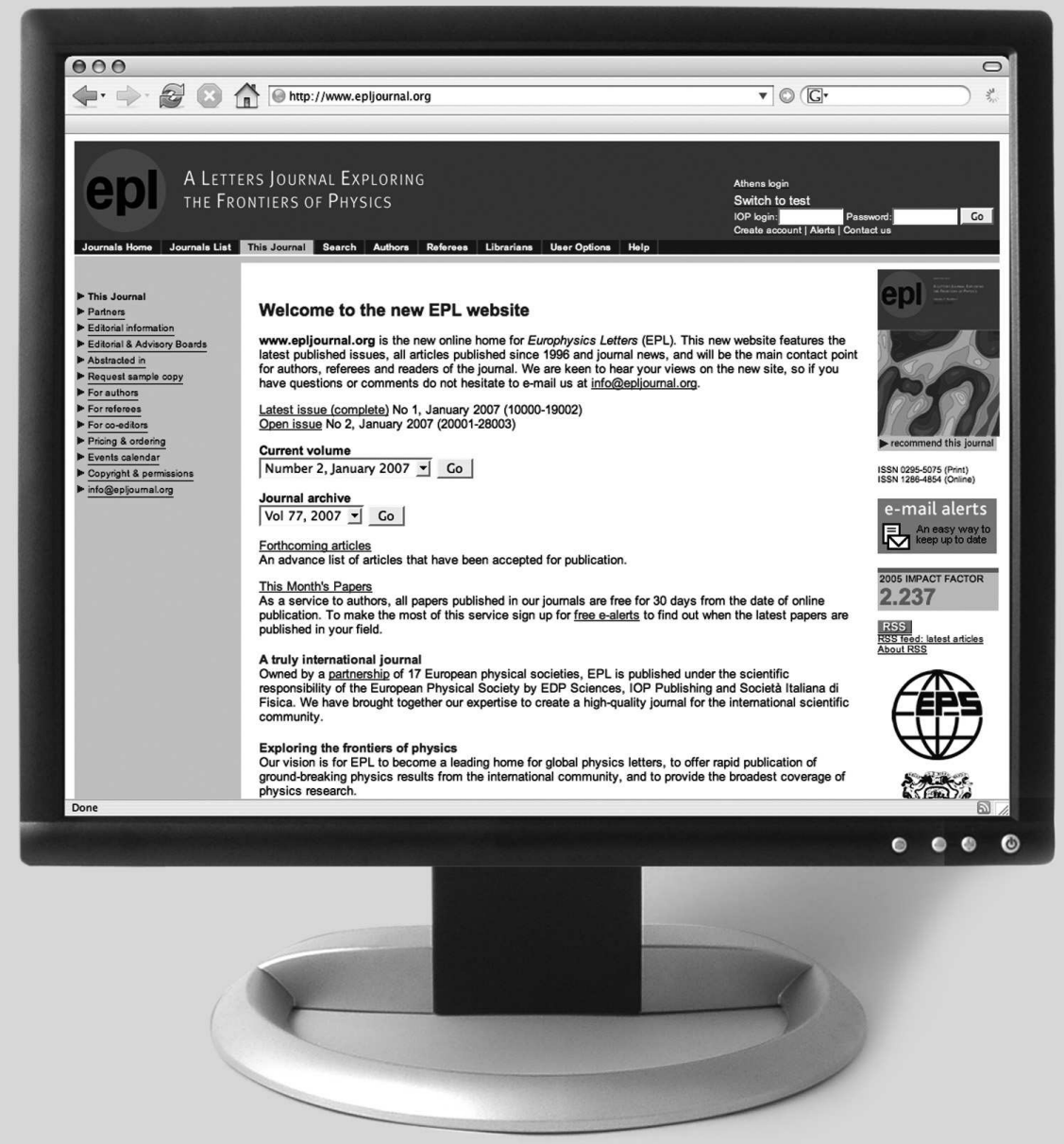

Take a look for the latest journal news and information on:

- reading the latest articles, free!

- receiving free e-mail alerts

- submitting your work to EPL 


\title{
Unstable dynamical systems: Delays, noise and control
}

\author{
J. G. $\operatorname{Milton}^{1(a)}$, J. L. CABrera ${ }^{2}$ and T. OhirA ${ }^{3}$ \\ ${ }^{1}$ Joint Science Department, The Claremont Colleges - Claremont, CA 91711, USA \\ ${ }^{2}$ Centro de Fúsica I. V. I. C. - Caracas 1020-A, Venezuela \\ ${ }^{3}$ Sony Computer Science Laboratories, Inc. - Tokyo, 141-0022, Japan
}

received 4 November 2007; accepted in final form 30 June 2008

published online 19 August 2008

PACS 87.18.Tt - Noise in biological systems

PACS 02.30.Ks - Delay and functional equations

PACS 02.50.Ey - Stochastic processes

\begin{abstract}
Escape from an unstable fixed point in a time-delayed dynamical system in the presence of additive white noise depends on both the magnitude of the time delay, $\tau$, and the initial function. In particular, the longer the delay the smaller the variance and hence the slower the rate of escape. Numerical simulations demonstrate that the distribution of first passage times is bimodal, the longest first passage times are associated with those initial functions that cause the greatest number of delayed zero crossings, i.e. instances where the deviations of the controlled variable from the fixed point at times $t$ and $t-\tau$ have opposite signs. These observations support the utility of control strategies using pulsatile stimuli triggered only when variables exceed certain thresholds.
\end{abstract}

Copyright (C) EPLA, 2008

Introduction. - As societies age, the morbidity and mortality associated with falls increases. In order to devise effective strategies to minimize the risk of falling it is first necessary to understand how balance is maintained. In physics, models for the maintenance of the upright posture have traditionally been formulated in terms of an inverted pendulum stabilized by continuous and time-delayed negative feedback [1-4]. Recent emphasis has been placed on two paradigms of human balance control, postural sway during quiet standing [5-9] and stick balancing at the fingertip [10,11]. Particularly puzzling has been the observation that the neural feedback in these situations is neither negative nor continuous! However, overlooked in these discussions are the possible beneficial effects of random perturbations (hereafter referred to as noise) on unstable systems [12-17]; especially when time delays are present $[10,11,18-21]$. Here we show that the interplay between noise, delay, and positive feedback makes possible a novel mechanism for balance control.

The experimental observation that the position controller for postural sway functions as a positive force feedback challenges long held beliefs that it should function as negative feedback (for reviews see [5-9]). During postural sway controlling forces arising from contractions of the calf muscles attempt to maintain the center of mass within the base of support formed by

(a) E-mail: jmilton@jsd.claremont.edu the area under the feet and the space between them. A mechano-reflex feedback systems generates force in relation to the movements of the center of mass. The mechanical stiffness of the muscle together with neural reflexes, principally the muscle stretch reflex, act togther as a negative feedback controller in which muscles act as a spring whose stiffness is dynamically set by reflex gain. Consequently, it was anticipated that forward sway would stretch (lengthen) muscles and lead to an increase in ankle torque. Recently this hypothesis was tested non-invasively by combining ultrasound with automated image analysis techniques to measure changes in the length of individual calf muscle fibers non-invasively during postural sway [8]. The observed changes are, on average, opposite to that expected for negative feedback: the average calf muscle fiber length shortens, not lengthens, on forward sway, and lengthens, nor shortens, on backward sway. Thus, on average, the controller behaves as positive feedback. Moreover, the control is not exerted continuously, but ballistically, i.e. in the form of brief, intermittent contractions [5].

The conclusion that position control feedback is positive also arises in studies of the closely related task of stick balancing at the fingertip [10,11,22-26]. During stick balancing the nervous system strives to minimize the vertical displacement angle. Using high-speed motion capture technologies it has been shown that the corrective movements occur intermittently and that $>95 \%$ of 
the time intervals between succesive corrective movements are shorter than the time delay [10]. Moreover, experiments involving both real [22] and virtual [23,24] stick balancing suggest that the stochastic reflex gain is tuned on average to the unstable side of the stability boundary. In other words, just as for postural sway, the feedback control for stick balancing is positive and the corrective movements are ballistic $[25,26]$.

How is it possible that positive feedback is part of a mechanism that controls an unstable state? One hypothesis, favored by neuroscientists [8,27], is that the nervous system is using a predictive control strategy. In other words corrections are based on anticipations of where the controlled variable will be at some point in the future. However, at least for stick balancing at the fingertip, this is unlikely since it has been shown that the corrective movements made by the fingertip are non-predictive [11]. In particular the changes in speed made by the fingertip are described by a Lévy distribution whose exponent is characteristic for a random foraging strategy.

An alternate hypothesis is that the balance feedback controller operates in a discontinuous, or switch-like, manner [2]: for small displacements the center of mass is allowed to "drift" (approximated as positive feedback control), corrective actions (negative feedback) occur only once displacement exceeds certain thresholds. Thus active control of balance is necessarily intermittent. This concept of discontinuous balance control is well supported by statistical analyses of the fluctuations of the center of pressure during postural sway [28], the switch-like properties of neural mechano-receptors [29] and motorneurons [30], and clinical observations of the ankle, hip and step strategies for maintaining balance as the magnitude and speed of perturbations increases [31]. Although models of this type for postural control have been proposed previously [2], little attention has been directed towards the role of the drift phase for determining the costs for this control. However, it is obvious that any mechanism that slows escape is of benefit since it reduces the number of activations of the negative feedback controller per unit time. Here we show that the required slowing of escape can come from the interplay between delay and noise. The positive time-delayed feedback reflects either a neural time-delayed feedback controller that is tuned near, or at, an edge of stability $[10,11]$ or because of the compliance of the muscle-elastic tissue complexes $[7,32]$.

We illustrate our hypothesis by studying the properties of an unstable delayed random walk, a model previously emphasized in models of postural sway [33,34] and stick balancing at the fingertip $[25,26]$. We show that the unstable delayed random walk exhibits two intrinsic properties that slow escape from its origin: firstly, the time delay, and secondly, the possibility that the dynamics can be "temporarily confined" near the origin.

Unstable delayed random walks. - We model the movements when the displacement is less than a threshold, $\left|X^{*}\right|$, as a 1-dimensional delayed random walk [33-35]: the walker takes a discrete step of unit length per unit time in a direction determined by conditional probabilities that depend on the position of the walker at some time, $\tau$, in the past. Models based on delayed random walks with an attractive origin provide an alternate and simpler approach for obtaining the stationary statistical properties of stochastic delay differential equations $[34,35]$ : it reduces the analysis to that of a higher-order Markov process. Delayed random walks are better suited for numerical simulations due to its discrete nature in space and time. Finally, insights into the transient properties can be obtained [34].

In order to derive a delayed random walk we generalize a position-dependent random walk so that the transition probability depends on its past state. One such represention is given by the following definitions (an alternate representation is given in [34]):

$$
\begin{aligned}
P(n, t+1)= & \sum_{m} g(m) P(n-1, t ; m, t-\tau) \\
& +\sum_{m} f(m) P(n+1, t ; m, t-\tau)
\end{aligned}
$$

where the position of the walker at time $t$ is $X(t), P(n, t)$ is the joint probability for the walker to be at $X(t)=n$ and $P(n, t ; m, t-\tau)$ is the joint probability such that $X(t)=n$ and $X(t-\tau)=m$ take place. $f(x)$ and $g(x)$ are transition probabilities for the walker coming from the right $(n+1)$ by taking a negative $(-1)$ step, or from the left $(n-1)$ by taking a positive $(+1)$ step such that

$$
f(x)+g(x)=1 .
$$

The definition (1) shows that these transition probabilities are dependent on the walkers position $\tau$ steps before. We assume that the random walk evolves on a symmetric (i.e. quadratic) potential surface (see Discussion), and hence $f$ and $g$ must be chosen to preserve this symmetry with respect to the origin, i.e.

$$
f(x)=g(-x)
$$

For the repulsive random walk we have

$$
f(x)<g(x) \quad(x>0) .
$$

It should be noted that the simple symmetric random walk can be recovered from (1) by choosing

$$
f(x)=g(x)=\frac{1}{2} \quad(\forall x) .
$$

Finally, since the force away from the origin linearly increases for the repulsive case, we choose $f, g$ to be

$$
f(x)= \begin{cases}\frac{1}{2}(1-2 d), & \text { if } x>a, \\ \frac{1}{2}(1-\beta x), & \text { if }-a \leqslant x \leqslant a, \\ \frac{1}{2}(1+2 d), & \text { if } x<-a,\end{cases}
$$




$$
g(x)= \begin{cases}\frac{1}{2}(1+2 d), & \text { if } x>a, \\ \frac{1}{2}(1+\beta x), & \text { if }-a \leqslant x \leqslant a, \\ \frac{1}{2}(1-2 d), & \text { if } x<-a,\end{cases}
$$

where $d$ is a constant such that $0 \leqslant d \leqslant 0.5$ and $\beta=2 d / a$. When $\tau=0$ this definition of transition probability makes the bias away from the origin to increase linearly with the distance from the origin with rate $\beta$, until it reaches $a$ : from that point on it is kept constant. We ignore the probability that the walker is outside the range $(-a, a)$ by setting $a=\left|X^{*}\right|$, where $X^{*}$ is an arbitrarily defined threshold (see below). These choices of $f, g$ are consistent with proposals that the fluctuations that arise in the setting of stick balancing reflect a critical balance between two opposing antagonistic forces [10,19,23,24].

Analysis. - For $t>0$ the position of the walker evolves while being influenced by its history at $\tau, X(t-\tau)$. The ballistic corrective movements are triggered whenever the displacement of the delay random walker exceeds the threshold, $\left|X^{*}\right|$. Thus, the first passage time, $L$, and its average $\hat{L}$, i.e. the average time that it takes a walker to cross an arbitrarily established threshold $\pm X^{*}$ are key parameters for understanding the properties of this control mechanism. Since the backward Kolmogorov formulation for such a random walk is not presently known, we cannot calculate $\hat{L}$ by, for example, backward propagation. Therefore we obtain a first approximation to $\hat{L}$ by calculating the variance. Then we use numerical simulations to investigate the nature of $L$.

Correlation functions. - The definition (1) can be extended to obtain an equation for the joint probability distribution [34]. Using similarly defined three-point probability distributions, we obtain for the repulsive delayed random walk

$$
\begin{aligned}
& P(n, t+1 ; \ell, t+1-u)= \\
& \quad \sum_{m} g(m) P(n-1, t ; \ell, t+1-u ; m, t-\tau) \\
& \quad+\sum_{m} f(m) P(n+1, t ; \ell, t+1-u ; m, t-\tau),
\end{aligned}
$$

where $P(n \pm 1, t ; \ell, t+1-u ; m, t-\tau)$ is the three-point joint probability distribution for the walker to be at $n \pm 1$, $\ell, m$ at, respectively, times $t, t+1-u$, and $t-\tau$, and $f$ and $g$ are given by (6), (7). We note that if we sum (8) with respect to $\ell$, we can recover (1).

The non-stationary time dependence of the correlation function

$$
K(u, t)=\langle X(t) X(t-u)\rangle,
$$

and hence the change in variance as a function of time

$$
\sigma^{2}(t)=K(0, t),
$$

can be obtained by multiplying (8) by $n \ell$ and summing over $n \ell$ where we have used the definition

$$
K(u, t)=\sum_{n, \ell} n \ell P(n, t ; \ell, t-u) .
$$

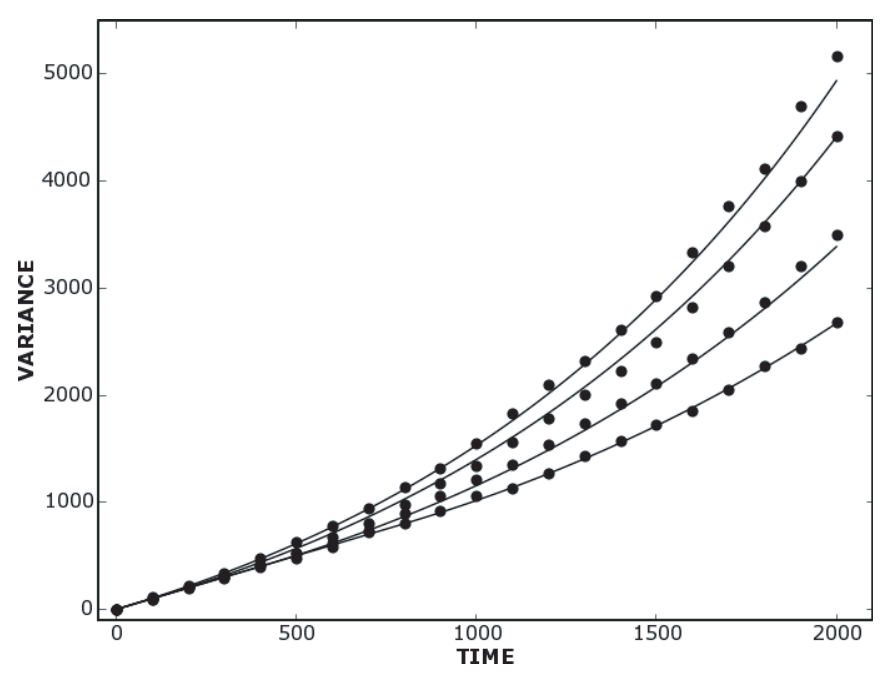

Fig. 1: Variance as a function of time for (17) for different values of the time delay: from top to bottom, $\tau=0, \tau=100, \tau=400$ and $\tau=800$. The variance predicted by numerical simulation of the coupled dynamical equations, (12)-(14), is given by the solid line and the values determined by numerical simulation of the repulsive delayed random walk with $f$ and $g$ given by, respectively (6) and (7), are indicated by $\bullet$ (mean value of 1000 realizations). Parameters: $d=0.01$ and $a=50$ with $\beta=2 d / a$.

In this way we obtain the following coupled dynamical equations for the correlation functions where we have identified $\beta$ with $\alpha$ in (17)

$$
\begin{aligned}
K(0, t+1)= & K(0, t)+1+2 \alpha K(\tau, t-\tau), \\
K(u, t+1)= & K(u-1, t+1) \\
& +\alpha K(\tau-(u-1), t+u-\tau),
\end{aligned}
$$

for $1 \leqslant u \leqslant \tau$, and

$$
\begin{aligned}
K(u, t+1)= & K(u-1, t+1) \\
& +\alpha K((u-1)-\tau, t+1),
\end{aligned}
$$

when $u>\tau$. As for the case when $\alpha<0$, the solution can be iteratively generated for the initial condition with the walker being kept fixed at the origin, i.e., $x(t)=\Phi_{0}(t)=0$, $t \in[-\tau, 0]$. This corresponds to the following boundary conditions:

$$
K(u, t)=0 \quad(-\tau \leqslant t \leqslant 0, \forall u) .
$$

Equations (12)-(14) were solved numerically. Figure 1 plots $K(0, t) v s$. time for various choices of $\tau$ for the boundary condition given by $(15)$. The predicted $K(0, t)$ is in excellent agreement with that obtained by simulating the repulsive delayed random walk using $f$ and $g$ given by, respectively, (6) and (7). Thus, to a first approximation $\hat{L}$ is expected to increase as $\tau$ increases.

First passage time. - Figure 2 demonstrates the stabilizing role of the initial function, $\Phi_{0}(s)$. In many cases, $\hat{L}$ for $\tau>0$ is longer than that for $\tau=0$. These 


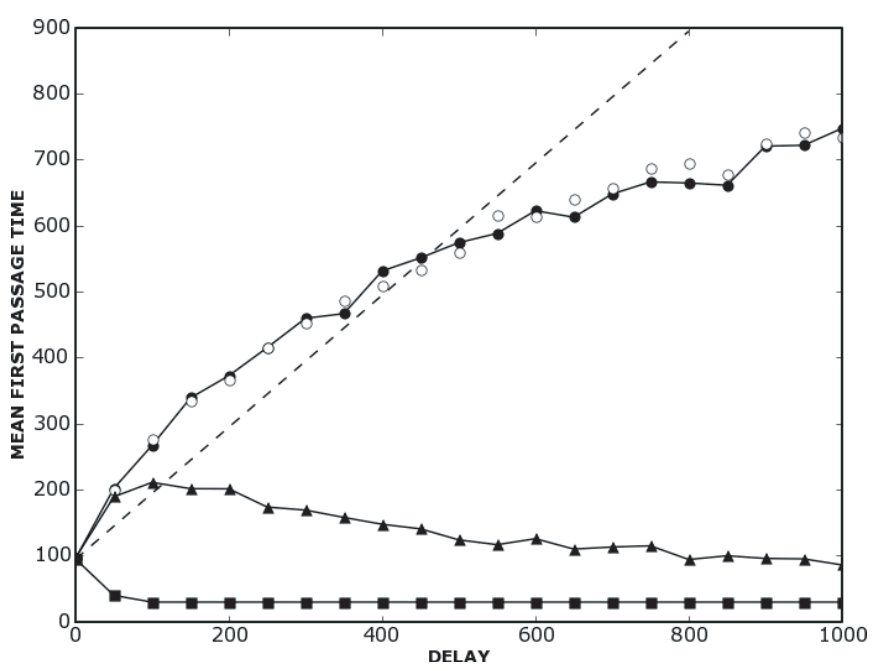

Fig. 2: The mean first passage time, $\hat{L}$, for a repulsive delayed random walk as a function of $\tau$ for four different choices of the initial function, $\Phi_{0}(t): 1$ ) a constant zero function (solid line, $\bullet$ ); 2) an initial function constructed by randomly choosing $+1,0,-1$ (०); 3) an initial function constructed from a simple random walk with $\tau=0$ (solid line, $\mathbf{\Delta}$ ); 4) a linear decreasing initial function with end points $x=\tau$ at $t=-\tau$ and $x=0$ at $t=0$ (solid line, $\boldsymbol{\square}$ ). In all cases $\Phi_{0}(0)=0$. The dashed line is equal to $\hat{L}_{\tau=0}+\tau$. For each choice of $\Phi_{0}(t), 500$ realizations were calculated with $X^{*}= \pm 30$ using (1) and (6), (7) with $d=0.4$ and $a=30$.

observations indicate that the escape of a delayed random walk can be prolonged by the walker's past history as contained in $\Phi_{0}(x)$. For some choices of $\Phi_{0}(x), \hat{L}$ increases monotonically as a function of $\tau$; for other choices $\hat{L}$ exhibits a more complex dependence on $\tau$. In contrast when $f>g$, the origin is destabilized by the delay for all choices of $\Phi_{0}(t)$, i.e. the stable states exist for non-zero walker positions [33].

In general, the proper choice of $\Phi_{0}(t)$ for an unstable dynamical system is not known. However, for the two paradigms which have motivated this study, namely stick balancing at the fingertip and postural sway during quiet standing, it can easily be arranged that $\Phi_{0}(t)=0$. For example, the stick at the fingertip can be held in the upright position by the other hand for a time $\tau$ and then released. Thus, for the remainder of this discussion we assume that $\Phi_{0}(t)=0$.

Figure 3a shows the distribution of first passage times, $N(L)$, when $\Phi_{0}(t)=0$. Clearly, when $\tau=700, N(L)$ is distinctly bimodal. The bimodal nature of $N(L)$ is not observed when $\tau=0$. In order to understand the nature of $N(L)$ it is helpful to consider the relative signs of $x(t)$ and $x(t-\tau)$ under the mapping defined by (1). If $x(t)$ and $x(t-\tau)$ have the same sign, then the delayed random walk will be biased towards the escape threshold since its transition probability to move in this direction is larger. On the other hand, if $x(t)$ and $x(t-\tau)$ have opposite signs, denoted herein as a delayed zero crossing, the effect will be
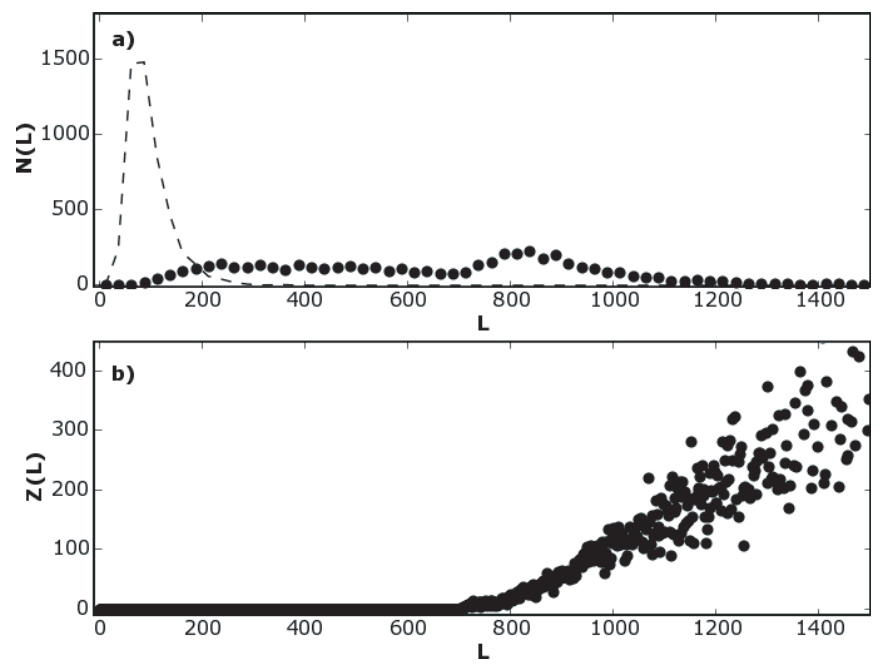

Fig. 3: Number of a) first passage times of length $L, N(L)$, and b) delayed zero crossings, $Z(L)$, as a function of the first passage time $L$. The repulsive delayed random walk is given for $\tau=0$ (dashed line) and $\tau=700(\bullet)$ with $d=0.4, a=30$, $\Phi_{0}(t)=0$ and $X^{*}=|30|$. It should be noted that for this choice of $\Phi_{0}(t)$ and $X^{*}, L$ is necessarily even and hence $Z(L)$ is only plotted for even $L$. There were no delayed zero crossings when $\tau=0$. Data points represent the averages for 5000 delayed random walker simulations with $\Phi_{0}(t)=0$.

to cause the walker on average to change direction away from threshold thus delaying the escape of the walker. Figure $3 \mathrm{~b}$ compares the number of delayed zero crossings, $Z(L)$, as a function of $L$ when $\tau=700$. As can be seen the second mode of the $N(L)$ is associated with the increased number of delayed zero crossings. Similar associations are obtained with those choices of the initial conditions that produced a monotonic increase in $\hat{L}$ with delay (see fig. 2).

Controlling instability. - The above observations suggest the advantage of using control strategies that employ pulsatile stimuli designed to increase the number of delayed zero crossings, which should increase $L$, and hence favor the slower pathways of escape. Obviously applying rapid perturbations with magnitude equal to the deviation of the current point from the fixed point, but of opposite sign, would stabilize the unstable point indefinitely. However, such control strategies would be both computationally and technically expensive to implement. Here we illustrate the feasibility of developing simple models for confining the dynamics of an unstable fixed point in the presence of delay by applying small perturbations less frequently.

Figure 4 compares two simple strategies for confining the random walkers near the origin. The first strategy applies periodically small amplitude corrections (20\% of the displacement from the origin). Although increasing the frequency of these periodic corrective movements shifts the survival curve to the right (compare $\boldsymbol{\Delta}$ and $\bullet$ in fig. $4 \mathrm{~b}$ ), all of the walkers eventually escape. 

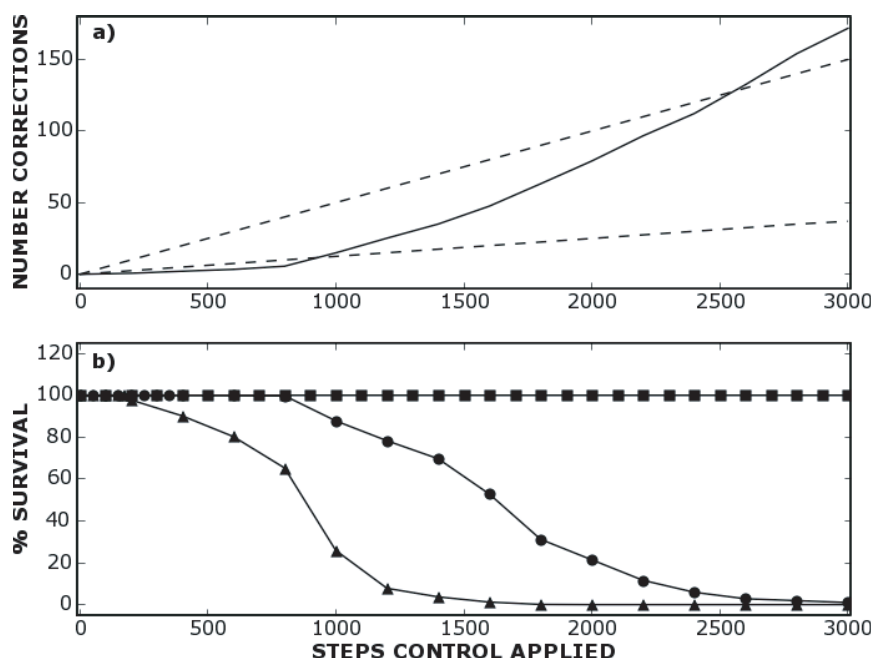

Fig. 4: a) Number of corrections made during the time interval in which the control strategy was applied. The dashed lines are for periodic corrections applied with a period of 80 steps (lower dashed line) or 20 steps (upper dashed line). The solid line gives the number of corrections using the threshold-type controller described in the text. In all cases the magnitude of the correction was $20 \%$ of the displacement from the origin. b) The percentage of 1000 delayed random walkers that survive, i.e. do not escape, during the time interval in which control was applied: periodic 20 steps control $(\bullet)$, periodic 80 step control ( $\mathbf{\Lambda})$, threshold-type control $(\boldsymbol{\square})$. For all simulations we used the repulsive random walk given by (1) and (6), (7) for $\tau=700, d=0.4, a=30,\left|X^{*}\right|=30,|\hat{X}|=20$, and $\Phi_{0}(t)=0$.

A slightly more intelligent control strategy can be devised by taking into account the bimodal nature of $N(L)$. In particular, we introduce a second threshold, $\hat{X}$, such that $0<|\hat{X}|<\left|X^{*}\right|$, and apply a corrective pulse whenever $|x(t)|>|\hat{X}|$. The secondary threshold $\hat{X}$ is introduced so as to discriminate between the slow and faster modes in $N(L)$. Control strategies of this types have been suggested both on the basis of mathematical models [36] and experimental observations $[2,28]$. In contrast to the periodic stimulation strategy, this threshold-type control strategy will confine the random walker near the origin as long as it is enforced. Moreover, for comparable control it is clear that methods based on the threshold-type model are more efficient.

Discussion. - It is unlikely that the nervous system can control posture using continuous negative feedback. Many of its sensory receptors exhibit switch-like properties, including bistability. Moreover, there are limitations for the use of continuous feedback in the setting of noise and delay [37]. An inherent problem is that of distinguishing those fluctuations that need to be acted upon by the controller from those which do not. This is because, by definition, there is a finite probability that an initial deviation away from a set point will be counter-balanced by one towards the set point just by chance. Too quick a response by a controller to a given deviation can lead to the phenomenon of "over control" leading to destabilization, particularly when time delays are appreciable. On the other hand, waiting too long runs the risk that the control may be applied too late to be effective. Thus, methods based on continous feedback control, e.g. [38,39], are not only anticipated to be very difficult to implement by the nervous system, but are also unlikely to be effective.

One way to overcome these problems is to use a switch-like, or discontinuous, feedback controller which is activated only when dynamical variables cross pre-set thresholds $[2,36,40]$. Switch-like controllers are well known to engineers and have the property that they are optimal when the control is bounded [40]. A familiar example of discontinuous feedback control arises in the thermostatic control of room temperature. It has been suggested that the ballistic corrective movements observed in balance control reflect "chattering", the dynamical signature of a switch-like feedback controller [9]. The beneficial effects of mechanisms that prolong the "wait phase", such as the effects of $\tau$ and those related to $\Phi_{0}(t)$ discussed here, would include transiently freeing up resources that could be used for other tasks [11] and energetic savings related to a less frequent use of the active controller per task. Our numerical simulations strongly support this possibility.

It is important to note that the attractive case for the delayed random walk model when $\tau=0$ corresponds to Ehrenfest's model for a discrete random walk in a harmonic potential [41,42]. By applying the same procedure for obtaining the Fokker-Planck equation from the Ehrenfest model for $\tau=0$ [41], we obtain, for $\tau \neq 0$,

$$
\begin{aligned}
\frac{\partial}{\partial t} P(x, t)= & \gamma \int_{-\infty}^{\infty} \frac{\partial}{\partial x} y P(x, t ; y, t-\tau) \mathrm{d} y \\
& +D \int_{-\infty}^{\infty} \frac{\partial^{2}}{\partial x^{2}} P(x, t ; y, t-\tau) \mathrm{d} y
\end{aligned}
$$

where $\gamma$ and $D$ are constants. On the other hand, starting with the delayed Langevin equation

$$
\mathrm{d} x(t)=-\alpha x(t-\tau) \mathrm{d} t+\mathrm{d} W
$$

where $\alpha>0$ and $W$ is the Wiener process, an equivalent expression for the Fokker-Planck equation has been obtained [43]. This correspondence carries over to the repulsive case. Indeed numerical simulations of (17) for $\alpha<0$ demonstrate qualitatively the same statistical properties as we report here for the corresponding delayed random walk including the bimodal distribution of first passage times (data not shown). Thus, the delayed random walk and the stochastic delay differential equation provide two different, but complimentary vantage points to understand the effects of $\Phi_{0}(s)$ on the evolution of this noisy and delayed dynamical system. In the case of the repulsive delayed random walk the dependence of the first passage times on $\Phi_{0}(s)$ arises because different paths in the past result in different paths in the future and in particular in numbers of delayed zero crossings. On the other hand, 
the key feature that dominates the behavior of (17) near the unstable fixed point is the co-existence of eigenvalues with negative and positive real parts [44,45]. Different choices of $\Phi_{i}(t)$ alter the relative contributions of the different eigenvalues to the solution; the application of brief pulses re-shuffles their contributions. Analogous situations in which eigenvalues with both negative and positive real parts co-exist arise when $\tau=0$ in the setting of a saddle point $[12,13,16,21]$ or Hopf bifurcation [46] and may, in part, explain the stabilizing effects of noise in these situations. Numerical simulations suggest that similar phenomena occur for $\tau \neq 0$ and account for the postponement of Hopf bifurcations [18] and delay-induced transient oscillations $[47,48]$.

The nervous system must necessarily contend with the effects of time delays and noise. It appears that, at least in the case of balance control, the nervous system has adopted a simple, but very robust control strategy. Namely, the system is simply allowed to drift (wait) until the controlled variable exceeds a threshold that initiates a corrective action (act). Our observation that such systems can be readily controlled using easily implemented control strategies may have far reaching implications.

\section{$* * *$}

We thank S. A. Campbell, D. Lai, G. B. ErmenTrout and M. C. MACKEY for useful comments. We acknowledge support from the William R. Kenan, jr. Foundation (JGM) NSF (Grant 0617072) (JGM, TO, JLC) and the IVIC (Grant IVIC-144) (JLC).

\section{REFERENCES}

[1] STÉPÁN G., Retarded Dynamical Systems: Stability and Characteristic Functions, Pitman Res. Notes Math. Ser., Vol. 210 (Wiley, New York) 1989.

[2] Eurich C. W. and Milton J. G., Phys. Rev. E, 54 (1996) 6681.

[3] Stépán G. and Kollár L., Math. Comput. Model., 31 (2000) 199.

[4] Yao W., Yu P. and Essex C., Phys. Rev. E, 63 (2001) 021902.

[5] Loram I. D. and Lakie M., J. Physiol., 540 (2002) 1111.

[6] Loram I. D. and Lakie M., J. Physiol., 545 (2002) 1041.

[7] Lakie M., Caplan N. and Loram I. D., J. Physiol., 551 (2003) 357.

[8] Loram I. D., Maganaris C. N. and Lakie M., J. Physiol., 564 (2005) 281.

[9] Bottaro A., Casadio M., Morasso P. G. and Sanguineti V., Hum. Mov. Sci., 24 (2005) 588.

[10] Cabrera J. L. and Milton J. G., Phys. Rev. Lett., 89 (2002) 158702.

[11] Cabrera J. L. and Milton J. G., Chaos, 14 (2004) 691.

[12] Kapista P. L. and Eksperim Z., Teor. Fiz., 21 (1951) 588.
[13] Bogdanoff J. L. and Citron S. J., J. Acoust. Soc. Am., 38 (1964) 447.

[14] Agudov N. V. et al., J. Phys. A, 37 (2004) 5279.

[15] Dettmann C. P., Keating J. P. and Prado S. D., J. Phys. A: Math. Gen., 37 (2004) L377.

[16] Graefe P. W. V., Arch. Appl. Mech., 35 (2004) 276.

[17] Kwon C., Ao P. and Thouless D. J., Proc. Natl. Acad. Sci. U.S.A., 102 (2005) 13029.

[18] Longtin A. et al., Phys. Rev. A, 41 (1990) 6992.

[19] Cabrera J. L., Physica A, 356 (2005) 25.

[20] Priplata A. et al., Phys. Rev. Lett., 89 (2002) 238102.

[21] Venkadesan M., Guckenheimer J. and ValeroCuevas F. J., J. Biomech., 40 (2007) 1653.

[22] Cabrera J. L. and Milton J. G., Nonlinear Stud., 11 (2004) 305.

[23] Bormann R. et al., Neurocomputing, 58-60 (2004) 517.

[24] Cabrera J. L. et al., Fluct. Noise Lett., 4 (2004) L107.

[25] Hosaka T. et al., Prog. Theor. Phys. Suppl., 161 (2006) 314.

[26] Cabrera J. L., Luciani C. and Milton J., Condens. Matter Phys., 9 (2006) 373.

[27] Menta B. and SchaAl S., J. Neurophysiol., 88 (2002) 942.

[28] Collins J. J. and De Luca C. J., Phys. Rev. Lett., 73 (1994) 764.

[29] Grigg P. and Greenspand B. J., J. Neurophysiol., 40 (1977) 1.

[30] Shapiro N. P. and Lee R. H., J. Neurophysiol., 97 (2007) 3948.

[31] Shumway-Cook A. and Woollacott M. H., Motor Control: Theory and Practical Applications (Williams \& Wilkins, New York) 2001.

[32] Proske U., Morgan D. L. and Gregory J. E., Prog. Neurobiol., 41 (1993) 705.

[33] Ohira T. and Milton J., Phys. Rev. E, 52 (1995) 3277.

[34] Ohira T. and Yamane T., Phys. Rev. E, 61 (2000) 1247.

[35] Ohira T. and Milton J., in Delay Differential Equations: Recent Advances and New Directions, edited by BALAChandran B., Kalmar-NAGY T. and Gilsinn D. (Springer-Verlag, New York) in press.

[36] Guckenheimer J., IEEE Trans. Autom. Control, 40 (1995) 321.

[37] Insperger T., IEEE Trans. Control Syst. Technol., 14 (2006) 974.

[38] Pyragus K., Phys. Lett. A, 170 (1992) 421.

[39] Yanchuk S. et al., Phys. Rev. E, 74 (2006) 026201.

[40] FlügGe-Lotz I., Discontinuous and Optimal Control (McGraw-Hill, New York) 1968.

[41] KaC M., Am. Math. Mon., 54 (1947) 369.

[42] Karlin S. and McGregor J., J. Appl. Probab., 2 (1965) 352.

[43] Frank T. D., Phys. Rev. E, 72 (2005) 011112.

[44] Wright E. M., J. Reine Angew. Math., 194 (1955) 66.

[45] Hayes N. D., J. London Math. Soc., 25 (1950) 226.

[46] Mackey M. C., Longtin A. and Lasota A., J. Stat. Phys., 60 (1990) 1572.

[47] Pakdaman K., Grotta-Ragazzo C. and Malta C. P., Phys. Rev. E, 58 (1998) 3623.

[48] Grotta-Ragazzo C., Pakdaman K. and Malta C. P., Phys. Rev. E, 60 (1999) 6230. 